\title{
Small asteroids temporarily captured in the Earth-Moon system
}

\author{
Robert Jedicke ${ }^{1}$, Bryce Bolin ${ }^{2}$, William F. Bottke ${ }^{3}$, Monique Chyba ${ }^{4}$, \\ Grigori Fedorets ${ }^{5}$, Mikael Granvik ${ }^{5}$ and Geoff Patterson ${ }^{4}$ \\ ${ }^{1}$ University of Hawaii, Institute for Astronomy, 2680 Woodlawn Dr., \\ Honolulu, HI, United States \\ email: jedicke@hawaii.edu \\ ${ }^{2}$ Nice Observatory, Nice, France \\ email: bryce.bolin@oca.eu \\ ${ }^{3}$ Southwest Research Institute, Boulder, CO, United States \\ email: bottke@boulder.swri.edu \\ ${ }^{4}$ University of Hawaii, Dept. of Mathematics, Honolulu, HI, United States \\ email: chyba@hawaii.edu, gpatters.uh@gmail.com \\ ${ }^{5}$ University of Helsinki, Helsinki, Finland \\ email: mgranvik@iki.fi, grigori.fedorets@helsinki.fi
}

\begin{abstract}
We present an update on our work on understanding the population of natural objects that are temporarily captured in the Earth-Moon system like the 2-3 meter diameter, $2006 \mathrm{RH}_{120}$, that was discovered by the Catalina Sky Survey. We use the term 'minimoon' to refer to objects that are gravitationally bound to the Earth-Moon system, make at least one revolution around the barycenter in a co-rotating frame relative to the Earth-Sun axis, and are within 3 Earth Hill-sphere radii. There are one or two 1 to 2 meter diameter minimoons in the steady state population at any time, and about a dozen larger than $50 \mathrm{~cm}$ diameter. 'Drifters' are also bound to the Earth-Moon system but make less than one revolution about the barycenter. The combined population of minimoons and drifters provide a new opportunity for scientific exploration of small asteroids and testing concepts for in-situ resource utilization. These objects provide interesting challenges for rendezvous missions because of their limited lifetime and complicated trajectories. Furthermore, they are difficult to detect because they are small, available for a limited time period, and move quickly across the sky.
\end{abstract}

Keywords. meteors, meteoroids; minor planets, asteroids; Moon; planets and satellites: general; surveys

\section{Introduction}

The Earth is orbited by small, natural, temporarily-captured, geocentric objects (Granvik et al., 2012) that could make excellent targets for scientific study and in-situ resource utilization (ISRU) demonstrations (Granvik et al., 2013). Some of these temporarilycaptured objects make at least one revolution around Earth in a co-rotating frame where the line connecting Earth and the Sun define the coordinate system (temporarilycaptured orbiters, TCO). The others, temporarily-captured fly-bys (TCF), are also energetically bound in the Earth-Moon system but do not make at least one revolution around Earth in the same frame (Granvik et al., 2012; Fedorets et al., 2015). We often refer to TCOs as 'minimoons' and TCFs as 'drifters' to avoid the use of three letter acronyms (TLA) and to illustrate the idea that TCOs are actually irregular natural satellites while the TCFs slowly drift through the Earth-Moon system. It is not surprising that only one TCO has ever been positively identified, $2006 \mathrm{RH}_{120}$ (Kwiatkowski et al., 2009), because 


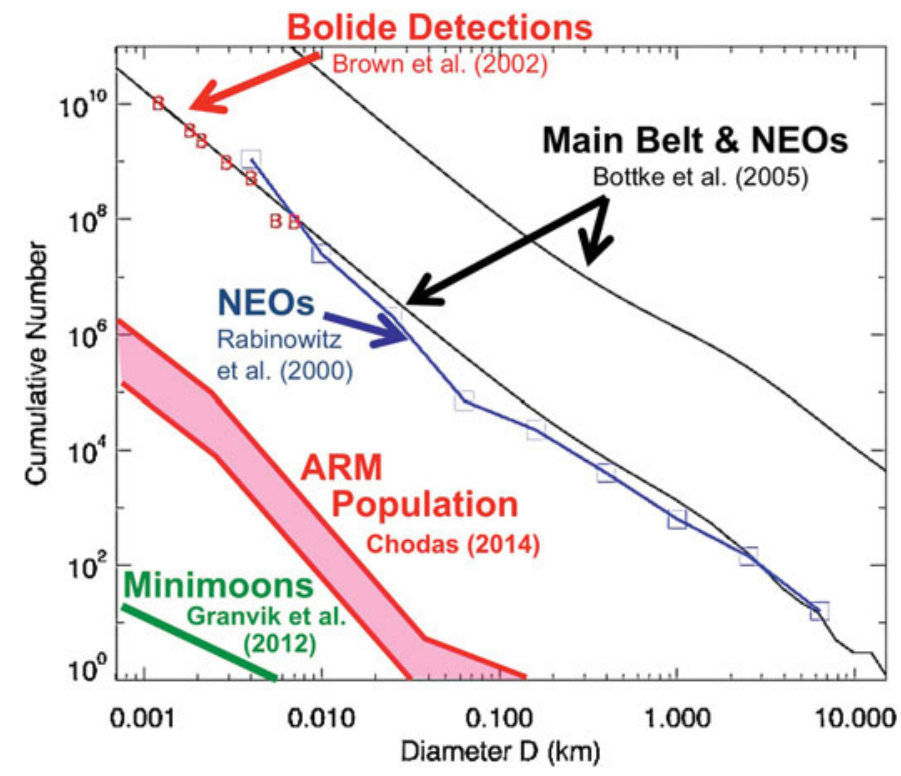

Figure 1. Size-frequency distribution of four populations of small objects in the inner solar system. The main belt feeds the NEO population, a subset of which is the ARM population, which in turn feeds the transient population of TCOs and TCFs.

their typical lifetime is only about 9 months and the largest objects in the steady state population are only about 1 to 2 meters in diameter.

\section{Origins}

Objects can become temporarily-captured in the Earth-Moon system only after running a gauntlet of improbable dynamical transitions (Fig. 1). Most of them originate as fragments of a collision between larger asteroids in the main belt between Mars and Jupiter. The orbits of the small fragments typically gradually evolve inwards or outwards in semi-major axis due to the persistent thermal reaction force known as the Yarkovsky effect. Eventually, a fragment will enter a region of orbital element space dominated by a mean-motion or secular resonance with the giant planets. These resonances typically have the effect of pumping up an objects' eccentricity until it's orbit has a perihelion of $<1.3 \mathrm{au}$ at which point it becomes known as a near-Earth object (NEO). The NEOs have characteristic lifetimes of only a few million years as their high eccentricity orbits typically eventually result in them striking the Sun, Jupiter, a terrestrial planet (notably Earth), or being ejected entirely from our solar system. A tiny fraction of the NEOs on low inclination orbits suffer a close approach with Earth, having the effect of removing the object from its giant planet resonance and, perhaps, reducing the orbital eccentricity to the level at which it becomes a desirable target for NASA's Asteroid Retrieval Mission (ARM; Chodas, 2014). These objects are desirable spacecraft rendezvous targets because their Earth-like orbits with semi-major axes of about $1 \mathrm{au}$, and close-to-zero eccentricities and inclinations make them accessible with small $\Delta v$ trajectories. Finally, a very small fraction of objects on Earth-like orbits pass through Earth's Hill sphere at slow enough speed and at the correct distance and direction to enable capture in the EarthMoon system within the n-body gravitational potential of the solar system (we note that geocentric capture of heliocentric objects is impossible in the circular restricted 3-body 


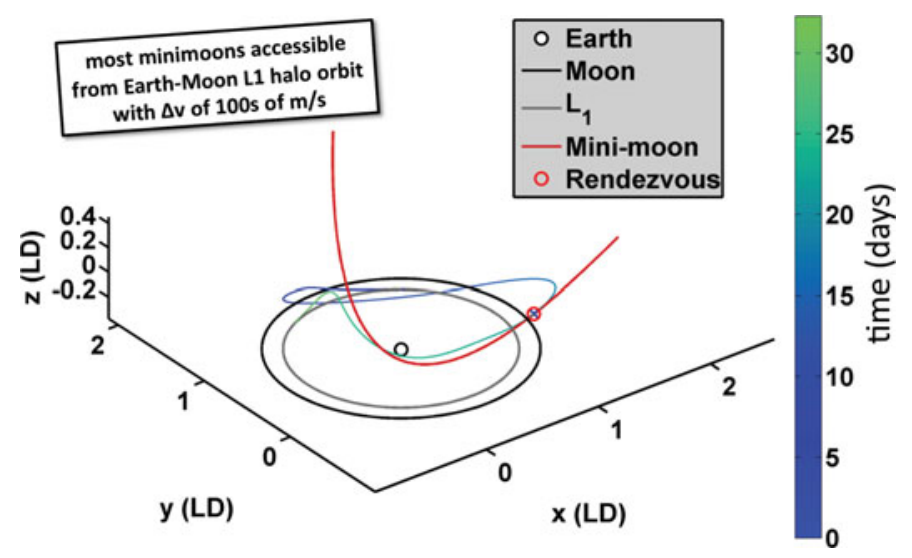

Figure 2. An example rendezvous (multicolored line with colour bar on right) between a spacecraft originally hibernating in an Earth-Moon L1 halo orbit and a minimoon (red line).

Sun-Earth-object system). The minuscule probability of running this dynamical gauntlet requires a large number of objects for even one to be temporarily captured. Thus, Granvik et al. (2012)'s simulations suggest that the largest objects in the TCO \& TCF steady state population is about 1 to 2 meters in diameter. The few-meter $2006 \mathrm{RH}_{120}$ was a somewhat unlikely outlier event that probably occurs about once a decade. About $1 \%$ of the TCOs' orbits evolve into Earth-impacting orbits and become meteors and, perhaps, meteorites.

An additional TCO creation mechanism is the launching of lunar regolith during an asteroid impact. Some of this material can escape lunar gravity, achieve selenocentric, geocentric, or heliocentric orbit, and almost half of the low-speed material that achieves heliocentric orbit eventually impacts Earth on a 50,000 year timescale (Gladman et al., 1995; Gladman, Burns, and Lee, 1995). Harris and D'Abramo (2015) suggest that many, it not most, of the meter-scale objects on Earth-like orbits could be former lunar ejecta that are, arguably, not as interesting for science or ISRU studies as main belt material. Thus, the relative contribution of lunar ejecta and main belt material to the TCO population is as yet unknown.

\section{Opportunities}

We think that the population of temporarily-captured geocentric objects provides several interesting scientific and engineering opportunities if the objects can be discovered in a methodical manner. Like a river that delivers gold from the mountains to the valley, the TCOs and TCFs may be fragments of the main belt delivered to cis-lunar space by solar system dynamics. Collisional, thermal, and dynamical processes have mined and transported main belt material directly to us and emplaced them in accessible, low $\Delta v$, geocentric orbits.

The 9 month average TCO lifetime makes it impossible to follow the normal mission protocol of planning a mission to a known target a decade in advance. Instead, we envision a rendezvous spacecraft in a 'parking' orbit around the Earth-Moon or Earth-Sun L1 or L2 points that hibernates until a suitable geocentric target becomes available and is then re-directed for rendezvous. We have shown that a large fraction of the TCOs are accessible from an Earth-Moon L1 or L2 halo orbit with $\Delta v$ s on the order of 100s of meters/sec (Fig. 2; Patterson (2015)) and also that the upcoming Large Synoptic Survey Telescope (LSST; Ivezic et al. (2008)) will identify dozens of TCOs and TCFs per year, a rate 
that should be sufficient to allow ample rendezvous target opportunities (Fedorets et al., 2015; Bolin et al., 2014). Rendezvous with meter-scale asteroids provides opportunities for direct interaction with material typical of asteroid surfaces. For comparison, NASA's ARM mission concept (Chodas, 2014) plans to retrieve a several-meter-scale asteroid from the surface of a heliocentric NEO and return it to lunar orbit for future science and engineering studies. All the scientific and engineering arguments in favor of the ARM mission would seem to apply to a mission to a geocentric asteroid with the added advantage that multiple TCOs and TCFs are available on a 9 month time scale. An even more ambitious plan could be to return a TCO or TCF to Earth. A single $0.5 \mathrm{~m}$ diameter object might provide of order $100 \mathrm{~kg}$ of pristine meteoroid unaffected by passage through Earth's atmosphere or weathering on Earth's surface. Alternatively, these objects could be used for testing ISRU options on real objects under real conditions in cis-lunar space.

Regardless of whether a rendezvous, ISRU, or return mission are possible, there are several scientific questions that can be explored with remote optical, infrared, and radar observations of small natural geocentric objects. First, and perhaps most interesting, is the measurement of their density using the area-to-mass ratio technique (e.g. Micheli, Tholen \& Elliot, 2014). It is well known that large asteroids have high macro porosities that must be due to large voids in their interiors but the scale at which the voids become significant is unknown. Are the boulder-size TCOs monolithic or heterogeneous, like $2008 \mathrm{TC}_{3}$, the only asteroid to be observed before it struck Earth and then have meteorite fragments recovered on the surface (Jenniskens et al., 2009)? Second, the Granvik et al. (2012) TCO model only accounts for gravitational dynamics and assumes that the size-frequency distribution of the meter-scale meteoroids matches that derived from meteor observations. So differences between the observed TCO orbit and size-frequency distribution are likely due to weak non-gravitational forces that act on the objects as they are delivered from the main belt into Earth orbit. Third, the rotation rates of small meteoroids are essentially unknown and the distribution of rotation rates alone could provide an indication of whether the objects are monolithic or heterogeneous. Finally, since about $1 \%$ of TCOs become meteors and some of them will create meteorites, it is likely that once 100s of TCOs are known it will be possible to trace an asteroid from space, through the atmosphere, and pick up its remnants on the surface. Connecting remote observations of a parent body, with targeted observations of the meteor phase, to lab-based studies of the constituent meteorites, is a scientifically rich opportunity as currently represented by the singular case of $2008 \mathrm{TC}_{3}$ (Jenniskens et al. (2009)).

\section{References}

Bolin, B., Jedicke, R., Granvik, M., Brown, P., Howell, E., Nolan, M. C., Jenniskens, P., Chyba, M., Patterson, G., \& Wainscoat, R. 2014, Icarus, 241, 280

Chodas, P. 2014, Asteroids, Comets, Meteors 2014, 94

Fedorets, G. \& Granvik, M. 2014, AAS/Division for Planetary Sciences Meeting Abstracts, 46, \#403.05

Fedorets, G., Granvik, M., Jones, L., \& Jedicke, R. 2015, IAU General Assembly, 22, 57052

Gladman, B. J., Burns, J. A., Duncan, M. J., \& Levison, H. F. 1995, Icarus, 118, 302

Gladman, J. B., Burns, A. J., \& Lee, P. 1995, Meteorite - The International Quarterly of Meteorites and Meteorite Science, 1, 16

Granvik, M., Jedicke, R., Bolin, B., Chyba, M., \& Patterson, G. 2013, Asteroids: Prospective Energy and Material Resources, 151

Granvik, M., Vaubaillon, J., \& Jedicke, R. 2012, Icarus, 218, 262

\& Harris, A. W. and D'Abramo, G. 2015, Icarus, 257, 302

Ivezic, Z., and 26 colleagues 2008, Serbian Astronomical Journal, 176, 1 
Jenniskens, P., and 34 colleagues 2009, Nature, 458, 485

Kwiatkowski, T., and 14 colleagues 2009, Astronomy and Astrophysics, 495, 967

Micheli, M., Tholen, D. J., \& Elliott, G. T. 2014, The Astrophysical Journal, 788, L1

Patterson, G. 2015, Ph.D. Thesis, University of Hawaii 